\title{
Unemployment Benefits and Unemployment
}

\author{
William Beranek, David R. Kamerschen* \\ Department of Economics, Terry College of Business, The University of Georgia, \\ Athens, Georgia \\ E-mail: "davidk@uga.edu
}

Received May 4, 2011; revised June 28, 2011; accepted July 23, 2011

\begin{abstract}
This paper seeks to provide a simpler explanation of the Match Quality Hypothesis (MQH). For the less mathematically inclined, it avoids formal analysis and yet derives the relevant implications, i.e., if unemployed workers currently collecting unemployment benefits are given more benefits, both the average period of unemployment duration increases as well as the level of unemployment. To produce these effects, only one person behaving in this manner is required. We cite recent evidence supporting these implications. Examined are implications of this theorem for both U.S. and European regions where, in some cases, voluntarily unemployed workers are eligible for unemployment benefits. We question the importance of the notion that generous unemployment benefits that intensify searches for better jobs, and hence prolonged job searches, ultimately yield societal benefits.
\end{abstract}

Keywords: Unemployment, Unemployment Benefits, Match Quality Hypothesis

\section{Introduction}

Much formal analysis has focused intensively on the response of benefit-collecting unemployed workers to an increase in benefits. Mortensen [1] suggests that 1) the opportunity costs of leisure time, even though workers may be unemployed, 2) the intensity of the job search and, 3) reservation wage prices play key roles in explaining duration of unemployment, and the level of steady-state unemployment. His model finds that the effect on unemployment duration of a benefit extension is ambiguous. However, numerous other writers, including Card and Levine [2], and Lavile, van Ours, and Zewimuller [3], consistent with earlier studies, predict a positive response in duration of unemployment and a higher steady state level of unemployment. In a related effort, Carling, Edin, Hackman, and Holmilund [4] hypothesize that the probability of the worker obtaining employment increases as the expiration of unemployment benefits expires. They find evidence to support this view. Our literature review is deliberately incomplete citing, for the most part, only those references that are immediately relevant to the MQH.

There is need, however, for a simpler, less formal, yet rigorous approach to explaining how a more generous benefit translates into a longer period of unemployment duration, and higher unemployment among those col- lecting benefits. In this effort, we also compare the effects of U.S. procedures of distributing benefits with the more liberal European style. In addition, we show that as few as one benefit collector responding to this stimulus of a benefit increase is sufficient to produce these consequences.

In sum, when faced with a jump in benefits, some unemployed people will tend to become more selective in the choice of a new job, a motive that focuses on enhancing the quality of the job. Whether the unemployed actually become choosier is, of course, an empirical question.

Increasing benefits can serve an important humanitarian aim among needy groups. However, there are those among the unemployed for whom such benefits can provide reduced incentives towards seeking immediate employment. Among these recipients, some may respond by not seeking employment at all. As shown above, investigators have sought to determine whether the number of those who become more selective is significant.

\section{The Hypothesis}

An increase in benefits to members of the unemployment group will tend to induce them to narrow their list of job targets. The reason for this assertion is that, after ranking possible job opportunities in order of preferences, and 
because of the additional unemployment benefits, the seeker will forego exploring inferior openings in favor of relatively more attractive ones. He can, and does, become more selective (Acemoglu and Shimer [5], Jovanovic [6], and Centeno [7]). These reduced options become his new set of opportunities, and in this sense, reduces incentives to search for jobs. In turn, the reduced set of job options implies a longer period of job search and a lengthening of the average period of unemployment. Because of lengthening unemployment spans, it also implies, all other things given, a tendency for the number unemployed to rise. In addition, an increase in benefits can intensify job-seeking efforts along with greater job selectivity.

\section{The Explanation}

A special case is sufficient to illustrate the notion. Suppose all unemployed persons receiving benefits obtain jobs in 30 days following the start of their benefits. With an extension of benefits, suppose they take an extra 30 days, or a total of 60 days. Assume also that the flow of new entrants into the unemployment pool remains constant. If so, counting from the day the extension is effective, for the next 30 days there will be no decreases in the unemployment rolls since people that were formerly leaving the rolls in 30 days now depart in 60 days. This means for the next 30 days the unemployment pool will rise to a higher level, reaching a new steady state. And this higher level will prevail as long as the rate of new entrants into the pool is equal to the rate at which they depart. The assumption of an additional 30 days of work search applies to new entrants as well. Even though this represents an extreme situation, it vividly illustrates what can happen.

Continue to assume that the rate of inflow into the unemployment pool remains constant. Suppose, as suggested by some writers (Lavile, Van Ours, and Zweimuller [3], that in response to the benefit increase some currently employed workers voluntarily join the ranks of benefit receivers, thus increasing the rate of new entrants to the pool. U.S. laws and institutions do not permit this response. To varying degrees, it is permissible in some European countries. In this case, should this result in an instantaneous response, the unemployment pool will increase more rapidly at first, reaching a new magnitude above the previous steady-state level, at which time it is assumed that the rate of inflow again becomes equal to the rate of outflow. The unemployment bulge is now due to two factors: Benefit recipients becoming more selective; and the voluntary response of currently employed workers to become benefit receivers. These results illustrate the dangers in attempting to draw inferences from mere changes in unemployment data.

Figure 1 provides a sketch of this process. The solid line from day 30 to day 60 captures the effect of the change in the number of unemployment compensation beneficiaries (NUCB) induced by an extension of the benefit period, the quality-job seeking effect. In the European culture, however, the solid line denotes the effects of the MQH on some European workers. (The same solid line denotes the hypothetical responses of both U.S. and European workers.) If, however, some currently employed European people leave their jobs to collect benefits, this produces the dashed line from day 30 to day 60, and beyond. In this case, previously employed workers join the unemployment pool at day 31 . For European workers, from day 31 and beyond, the dashed line is the sum of two effects: 1) the addition to the pool of benefit collectors induced to leave gainful work voluntarily in order to collect benefits but who also take 60 days to gain new employment. 2) The addition due to some members of the recipient pool extending their work search by 30 days, the MQH effect. If the European response among currently employed workers is repeated every 60 days, then the numbers of new benefit collectors always equal the numbers leaving the pool, i.e., those obtaining new jobs, and a so-called steady state is attained.

The response in unemployment due to workers behaving in this manner is remarkably sensitive. Let us rule out forward-looking workers who leave employment in response to an increase in benefits, as well as those who increase their enthusiasm for job-search. Regardless of country, it is remarkable that only one person among the unemployed with the MQH trait is required to increase the average duration of unemployment and the level of unemployment. There is, as it were, a bottleneck created by the temporary reduction to zero of the rate of outflow of workers from the pool of benefit recipients. However, as long as new entrants into the pool behave in accordance with the $\mathrm{MQH}$, and the inflow rate is equal to the rate of outflow, this bulge in the levels of benefit collec-

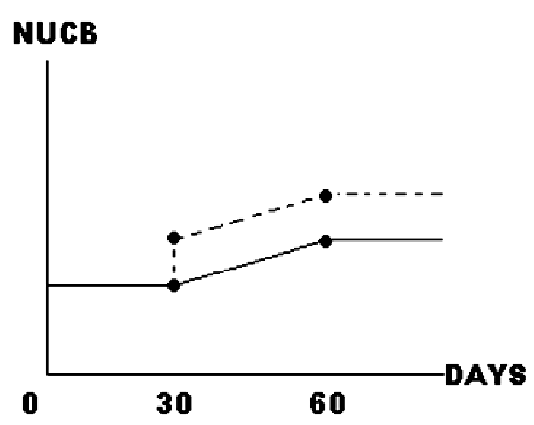

Figure 1. The behavior of NUCB. 
tors can be sustained indefinitely ${ }^{1}$.

Of course, if benefits are slashed, and if behavioral relations remain as assumed, the process is reversed. The average period of unemployment declines, inducing a reduction in the steady-state unemployment total.

\section{Further Implications}

The question is when benefits are initially granted, does not a response similar to the one outlined above take place? Yes, the same process unfolds and, all other things given, the average period of unemployment increases followed by an expansion in unemployment.

Implied by our analysis is the important fact that an increase in total unemployment does not signify an increase in the rate at which workers are being laid-off. This myth is common, especially among news commentators. In the same vein, an expansion of unemployment rolls does not imply decreases in the rate employers are hiring new workers. Such an easy-to-make misinterpretation appears to be widespread among journalists.

\section{Empirical Studies}

A rich literature exists on the effect of benefits on the duration of unemployment. See, for example, Moffitt and Mickelson [8], Moffitt [9], and Katz and Meyer [10] for studies of U.S. data, and Nickell and Layard [11] and Machin and Manning [12] for examinations of European data. The two most recent U.S. studies are Card and Levine [2] who found that, using New Jersey data, extension of benefits by 13 weeks leads to an increase of $7 \%$ in the number of recipients exhausting their regular (i.e., pre- extension benefits) and a 1 week increase in the average period of unemployment. Katz and Meyer [10], using a different sample, reported an increase in the duration of unemployment of 2 to 2.5 weeks.

Finally, employing Austrian data, Lavile, van Ours and Zweimuller [3] found that benefit increases lead to significant increases in the steady-state unemployment rate. They found that the most important factor leading to this increase is not the increased duration of unemployment among the existing jobless, but to the increase in incentives for currently employed workers to leave their jobs and receive benefits. The reader is cautioned not to

\footnotetext{
${ }^{1}$ A physical analogy may be helpful. Consider a washbasin with a constant rate of water inflow, and the same constant rate of outflow at the drain. As long as these equal rates of flows remain stationary, the water level in the basin will likewise remain stationary. Now suppose we plug the drain for a period of 10 minutes. Clearly, the water level will rise by an amount equal to the accumulated rates of inflow over the 10-minute interval. After the 10-minute span, the plug is removed, and if the two rates of flows are restored to their pre-plug rates, the new higher water level will be sustained indefinitely. The water level is analogous to total unemployment.
}

put too much credence on the comparative values of these U.S. and European estimates. The differences in institutions, labor laws, union policies, and employer policies across states and countries are so vast, making magnitude inferences hazardous.

\section{A Related Hypothesis}

People who take advantage of added benefits and obtain better jobs, it is suggested, may be more efficiently employed and hence of greater value to society (Centeno [7]). Unanswered are the questions of how to measure efficiencies and their magnitude. However, such benefits are potentially available anyway because job seekers have viable alternatives. Particularly, the seeker may accept a less satisfactory job while continuing the search for an improved position. Many people do just that. Consequently, society obtains these hypothesized added efficiencies in the normal functioning of the labor market without requiring a boost in unemployment compensation. In a related vein, university professors, even though gainfully employed, are notoriously open to exploring other opportunities, and their professional meetings provide, among other things, a perfect setting for such activities. Nevertheless, Centeno [7], using the length of a re-employed worker's subsequent job tenure as a proxy for job quality, finds evidence to support this belief. This proxy, however, is fraught with hazards since there are so many important variables that influence length of subsequent employment.

\section{Conclusions}

As noted by many writers, increases in unemployment benefits can provide reduced incentives for the jobless to seek jobs. The job seeker may increase his overall quality standards in demanding a new job, the Match-Quality Hypothesis. The seeker can, and does, become more selective. This, in turn, increases the duration of unemployment for those receiving benefits and, all other things given, increases the level of unemployment among those as well. Surprisingly, only one person with the $\mathrm{MQH}$ trait is needed to produce a slight bulge in unemployment duration, and hence in total unemployment. Available empirical studies provide evidence consistent with these predictions. In addition, these behavioral traits appear to be empirically valid regardless of the culture of the work force.

\section{Acknowledgements}

Professor Emeritus of Financial Economics and Jasper 
N. Dorsey Professor of Economics, respectively, University of Georgia. We are grateful to James A. Craft and Ronald Warren for helpful comments.

\section{References}

[1] D. T. Mortensen, "Unemployment Insurance and Job Search Decisions," Industrial and Labor Relations Review, Vol. 30, No. 4, 1977, pp. 505-517.

doi:10.2307/2523111

[2] D. Card and P. B. Levine, "Extended Benefits and the Durations of UI Spells: Endurance from the New Jersey Extended Beneficial Program,” Journal of Public Economics, Vol. 78, No. 1-2, 2000, pp. 107-138. doi:10.1016/S0047-2727(99)00113-9

[3] R. Lavile, J. Van Ours and J. Zewimuller, "How Changes in Financial Incentives Affect the Duration of Unemployment," Review of Economic Studies, Vol. 73, No. 4, 2006, pp. 1009-1038. doi:10.1111/j.1467-937X.2006.00406.x

[4] K. Carling, P.-A. Edin, A. Hackman and B. Holmlund, "Unemployment Duration, Unemployment Benefits and Labor Market Programs in Sweden,” Journal of Public Economics, Vol. 59, No. 3, 1996, pp 313-334. doi:10.1016/0047-2727(95)01499-3

[5] D. Acemoglu and R. Shimer, "Productivity Gains from Unemployment Insurance,” European Economic Review,
Vol. 44, No. 7, 2000, pp. 1195-1224. doi:10.1016/S0014-2921(00)00035-0

[6] B. Jovanovic, "Job Matching and the Theory of Turnover,” Journal of Political Economy, Vol. 87, No. 5, 1979, pp. 972-990. doi:10.1086/260808

[7] M. Centeno, "The Match Gains from Unemployment Insurance,” Journal of Human Resources, Vol. 34, No. 3, 2004, pp. 839-863. doi:10.2307/3559000

[8] R. Moffit, "Unemployment Insurance and the Distribution of Unemployment Spells,” Journal of Econometrics, Vol. 28, 1995, pp. 85-101.

[9] R. Moffit and W. Nicholsen, "The Effect of Unemployment Insurance on the Unemployed; the Case of Federal Supported Benefits," Review of Economics and Statistics, Vol. 64, No. 1, 1982, pp. 1-11. doi:10.2307/1937937

[10] L. F. Katz and B. D. Meyer, "Unemployment Insurance, Recall Expectationsand Unemployment Outcomes,” Quarterly Journal of Economics, Vol. 105, No. 4, 1990, pp. 973-1002. doi:10.2307/2937881

[11] S. Nickell and R. D. Layard, "Labor Market Institutions and Economic Performance,” In: O. C. Ashenfelter and D. Card, Eds., Handbook of Labor Economics, Vol. 3, Part C, 1999, pp. 3029-3084.

[12] S. Machin and A. Manning, "Causes and Consequences of Longterm Unemployment in Europe,” In: O. C. Ashenfelter and D. Card, Eds., Handbook of Labor Economics, Vol. 3, Part C, 1999, pp. 3085-3139. 